Aaron Lim, Centre for Mathematical Biology, Oxford University, Oxford, UK

Sunetra Gupta, Department of Zoology, Oxford University, Oxford, UK

Philip Maini, Centre for Mathematical Biology, Oxford University, Oxford, UK

\title{
HTLV-I Infection: A Dynamic Struggle Between Viral Persistence and Host Immunity
}

Human T-lymphotropic virus type I (HTLV-I) is a persistent human retrovirus characterised by life-long infection and risk of developing one of two major, clinically independent diseases: adult T-cell leukaemia/lymphoma (ATL), an aggressive blood cancer, and HAM/TSP, a progressive neurological and inflammatory disease. Infected individuals typically mount a large, chronically activated $\mathrm{CD} 8^{+}$cytotoxic T-lymphocyte (CTL) response against HTLV-I-infected cells, but ultimately fail to effectively eliminate the virus. Moreover, identification of determinants to disease manifestation has thus far been elusive.

A key issue in current HTLV-I research is to better understand the dynamic interaction between persistent infection by HTLV-I and virus-specific host immunity. Recently, Asquith and Bangham [1] have proposed an experimental hypothesis for the persistence of HTLV-I in vivo which has motivated the formulation of a mathematical model by Li and Lim [2] that illustrates the balance between latency and activation in the target cell dynamics of the viral infection. We present an extension of this previous model that incorporates the role of a constantly changing anti-viral immune environment mediated by HTLV-I-specific CTLs. The resulting model is a four-dimensional system of ordinary differential equations that describes the dynamic interactions among viral expression, infected target cell activation, and the HTLV-I-specific CTL response.

We have identified a sharp threshold parameter, the basic reproduction number for viral infection $R_{0}$, which completely characterises the global behaviour of solutions to the model: if $R_{0}<1$, the infection is cleared; if $R_{0}>1$, the infection is chronic. The global stability of the respective equilibrium state in each of the two cases for $R_{0}$ has been shown by constructing appropriate Lyapunov functions.

Having established the global dynamics of the model, we set out to address biologically relevant questions in the case of chronic HTLV-I infection. In particular, we focussed on three issues: (i) How is the virus able to evade CTLmediated elimination and not only establish, but also maintain, infection? (ii) What role do CTLs play in controlling the virus? (iii) Do our results provide insights to the development of HTLV-I-associated disease?

Using bifurcation analysis and computational methods, we explored the roles of certain key parameters on the outcome of the infection dynamics. The results of our investigation offer important insights to the evolution of viral persistence and proposes a hypothesis for pathogenesis.

\section{References}

[1] B. Asquith and C. R. M. Bangham (2008) How does HTLV-I persist despite a strong cell-mediated response?, Trends in Immunol., 29(1):4-11.

[2] M. Y. Li and A. G. Lim (2011) Modelling the role of Tax expression in HTLV-I persistence in vivo, Bull. Math. Biol., 73(12):3008-3029. 